Supplement 2, Vol. 14,

December 2007

\section{Medical Pluralism, Equity, Holism}

Interdisciplinary Research Perspectives on Integrative Medicine

Editors

Florica Marian, Bern

Harald Walach, Northampton 


\section{Komplementärmedizin \\ Wissenschaft • Praxis • Perspektiven}

Vol. 14, Supplement 2, Dezember 2007

Editorial

1 Medical Pluralism, Equity and Holism Marian, F. (Bern); Walach, H. (Northampton)

Original Article

2 Complementary Medicine: Equity Issues in Evaluation and Policy-Making

Marian, F. (Bern)

Essay

10 Medical Pluralism: Global Perspectives on

Equity Issues

Marian, F. (Bern)

Original Article

19 Exploring Different Dimensions of Holism: Considerations in the Context of an Evaluation of Complementary Medicine in Primary Care Marian, F. (Bern)

II Imprint

28 Guidelines for Authors
Band 14, Supplement 2, December 2007

Editorial

1 Pluralismus in der Medizin, Gerechtigkeit und Ganzheitlichkeit

Marian, F. (Bern); Walach, H. (Northampton)

Originalarbeit

2 Komplementärmedizin: Gerechtigkeitsfragen im Zusammenhang mit Evaluation und Gesundheitspolitik Marian, F. (Bern)

Essay

10 Pluralismus in der Medizin: Globale Perspektiven zur Gerechtigkeit

Marian, F. (Bern)

Originalarbeit

19 Untersuchung verschiedener Dimensionen von Ganzheitlichkeit: Überlegungen im Rahmen einer Evaluation der Komplementärmedizin in der Grundversorgung

Marian, F. (Bern)

II Impressum

U3 Hinweise für Autoren (3. Umschlagseite)

\section{KARGER}

Fax +497614520714

E-mail Information@Karger.de

www.karger.com 